\title{
Combined effect of proton-pump inhibitors and other drugs with regard to lower gastrointestinal tract bleeding with special reference to low-dose aspirin
}

\author{
Tomoyuki Kawada ${ }^{1}$
}

Received: 15 September 2015/ Accepted: 16 September 2015/Published online: 6 November 2015

(C) Japanese Society of Gastroenterology 2015

Nagata et al. [1] investigated the effects of proton-pump inhibitors (PPIs) and the effects of their interactions with several drugs, including low-dose aspirin, with regard to the risk of lower gastrointestinal tract bleeding. They conducted a case-control study, and the adjusted odds ratios for lower gastrointestinal tract bleeding were estimated by use of a logistic model. PPI use did not result in any significant increase in the risk of lower gastrointestinal tract bleeding. In addition, the adjusted odds ratio of PPI use for lower gastrointestinal tract bleeding did not become significant for any concomitant therapy with nonsteroidal anti-inflammatory drugs, low-dose aspirin, clopidogrel, or warfarin. I have some queries regarding their study.

First, Miyake et al. [2] recently conducted a retrospective cohort study that confirmed the combination of low-dose aspirin and PPIs increased the risk of lower gastrointestinal tract bleeding in patients with ischemic heart disease. The adjusted hazard ratio for concomitant use of PPIs was 6.55 (95\% confidence interval 2.01-21.32) in 548 patients using low-dose aspirin. I suspect that the wide range of the $95 \%$ confidence interval was caused by the limited number of patients with lower gastrointestinal tract bleeding $(n=18)$, and the combination of low-dose aspirin and PPIs should be used with caution because of the risk of lower gastrointestinal tract bleeding. In addition, the study design is different between the two studies, and discrepancies in the study outcomes should be confirmed by further study.

This comment refers to the article available at doi:10.1007/s00535015-1055-2.

Tomoyuki Kawada

kawada@nms.ac.jp

1 Department of Hygiene and Public Health, Nippon Medical School, 1-1-5 Sendagi, Bunkyo-Ku, Tokyo 113-8602, Japan
Second, there are two types of logistic regression analysis, and conditional logistic regression analysis can be applied to a case-control study with a sufficient number of controls. If they conducted individual matching and they used an unconditional logistic model, the study outcomes would become conservative estimates. Namely, no statistical significance can be caused partly by the statistical procedure. As Nagata et al. had a sufficient number of controls, they should have used the appropriate logistic model for their study.

Finally, there are several causes of lower gastrointestinal tract bleeding, and about half of the cases were due to diverticular bleeding. I recommend Nagata et al. conduct a sensitivity analysis with special reference to the main cause of lower gastrointestinal tract bleeding to simplify the combined effect of PPIs and several drugs, including lowdose aspirin. As their study cannot guarantee the safety of the combination of PPIs and nonsteroidal anti-inflammatory drugs, low-dose aspirin, clopidogrel, or warfarin, further study is recommended for patients using PPIs.

Compliance with ethical standards

Conflict of interest The author declares that he has no conflict of interest.

\section{References}

1. Nagata N, Niikura R, Aoki $\mathrm{T}$, et al. Effect of proton-pump inhibitors on the risk of lower gastrointestinal bleeding associated with NSAIDs, aspirin, clopidogrel, and warfarin. J Gastroenterol. 2015. doi:10.1007/s00535-015-1055-2.

2. Miyake K, Akimoto T, Hanada Y, et al. Proton pump inhibitors are associated with lower gastrointestinal tract bleeding in low-dose aspirin users with ischaemic heart disease. Dig Liver Dis. 2015; $47: 757-62$. 